\title{
Constant of Motion Identifying Excited-State Quantum Phases
}

\author{
Ángel L. Corps $\odot^{*}$ and Armando Relaño \\ Departamento de Estructura de la Materia, Física Térmica y Electrónica and Grupo Interdisciplinar de Sistemas Complejos (GISC), \\ Universidad Complutense de Madrid, Avenida Complutense s/n, E-28040 Madrid, Spain
}

(Received 26 March 2021; revised 26 July 2021; accepted 3 September 2021; published 24 September 2021)

\begin{abstract}
We propose that a broad class of excited-state quantum phase transitions (ESQPTs) gives rise to two different excited-state quantum phases. These phases are identified by means of an operator $\hat{\mathcal{C}}$, which is a constant of motion in only one of them. Hence, the ESQPT critical energy splits the spectrum into one phase where the equilibrium expectation values of physical observables crucially depend on this constant of motion and another phase where the energy is the only relevant thermodynamic magnitude. The trademark feature of this operator is that it has two different eigenvalues \pm 1 , and, therefore, it acts as a discrete symmetry in the first of these two phases. This scenario is observed in systems with and without an additional discrete symmetry; in the first case, $\hat{\mathcal{C}}$ explains the change from degenerate doublets to nondegenerate eigenlevels upon crossing the critical line. We present stringent numerical evidence in the Rabi and Dicke models, suggesting that this result is exact in the thermodynamic limit, with finite-size corrections that decrease as a power law.
\end{abstract}

DOI: 10.1103/PhysRevLett.127.130602

Introduction.-A quantum phase transition happens when an abrupt change in the ground state of a physical system is observed. The corresponding critical point is signaled by a nonanalyticity, and two different phases can be identified by equilibrium measurements [1]. Excitedstate quantum phase transitions (ESQPTs), a generalization of this phenomenon to excited states, have been the focus of intense research during the past years [2,3] (for a recent, excellent review, see [4]). ESQPTs give rise to a great variety of dynamical consequences, like huge decoherence [5,6]; singularities in quench dynamics [7-11], feedback control in dissipative systems [12], quantum work statistics [13], and localization [14]; symmetry-breaking equilibrium states [15,16]; universal dynamical scaling [17]; dynamical instabilities [18]; irreversibility without energy dissipation [19]; and reversible quantum information spreading [20]. They are somehow linked to thermal phase transitions $[21,22]$ and dynamical phase transitions [23,24]. They can be identified by their consequences in the classical [25] and semiclassical $[26,27]$ phase-space dynamics $[28,29]$, as in the singularities of the density of states [30]. Its signatures have been theoretically and experimentally observed in several physical systems [31-36], and its connections with quantum Lyapunov exponents have been explored [37-39]. However, no physical features of standard phase transitions have been identified yet.

In this Letter, we show that a typical feature of a large class of ESQPTs splits the spectrum into two different excited-state quantum phases. These are identified by an operator which is a constant of motion in just one of them (usually, below the ESQPT). This constant of motion signals to which part of the semiclassical phase space a given quantum state is attached and assigns it a conserved quantum number, with important thermodynamic consequences. We present general arguments and illustrate our findings with the paradigmatic Rabi (RM) [40,41] and the Dicke (DM) [42-46] models, discussing dynamical and thermodynamic consequences.

Constant of motion.-We start from the classical limit of a quantum system with Hamiltonian $H(\mathbf{x})$, where $\mathbf{x} \in \mathbb{R}^{2 \nu}$ accounts for all relevant canonical coordinates and $\nu \in \mathbb{N}$ is the number of classical degrees of freedom. ESQPTs are caused by fixed points $\mathbf{x}_{c}$ of the classical Hamiltonian flow, $\nabla H\left(\mathbf{x}_{c}\right)=0$, at a critical energy $E_{c} \equiv H\left(\mathbf{x}_{c}\right)$ [4]. The main result of this Letter is the following conjecture.

Suppose there exists a dynamical function $f(\mathbf{x})$ satisfying the following two properties: (i) $f\left(\mathbf{x}_{c}\right)=0$ and (ii) on one side of the transition (say, $E<E_{c}$ ), every trajectory verifies either $f(t) \equiv f(\mathbf{x}[t])<0$ or $f(t)>0, \forall t$, depending on the initial condition, but this is no longer true on the other side. Then, there exists a quantum operator, $\hat{\mathcal{C}} \equiv \operatorname{sgn}[\hat{f}(\hat{\mathbf{x}})]$, which is a constant of motion only in the first of these two phases, $E<E_{c}$.

Here, the sign of an operator $\hat{f}$ is defined $\operatorname{sgn}(\hat{f}) \equiv F \operatorname{sgn}(D) F^{-1}=F \operatorname{diag}\left[\operatorname{sgn}\left(\left\{d_{i}\right\}\right)\right] F^{-1}$, where $D$ is a diagonal matrix whose elements $\left\{d_{i}\right\}_{i}$ are the eigenvalues of $\hat{f}$ and $F$ is a matrix whose columns are the eigenvectors of $\hat{f}$. Hence, the operator $\hat{\mathcal{C}}$ has only two eigenvalues \pm 1 and, therefore, represents a $\mathbb{Z}_{2}$ symmetry in this phase. Notwithstanding, it is unrelated to any exact discrete symmetry of a given model, and, thus, it is not linked to spontaneous symmetry breaking observed in some phase transitions. As representative examples of 
systems fulfilling the conditions for the above conjecture, we quote the Lipkin-Meshkov-Glick model, the Rabi and Dicke models, spinor Bose-Einstein condensates and Bose mixtures in a double-well potential, the coupled top, and the two-fluid Lipkin model $[2,5,6,12,15,22,25-28,43,47-$ 50]. In all these systems, there is an ESQPT at $E=E_{c}$ below which the classical phase space is split into disconnected wells. Then, $\hat{\mathcal{C}}$ indicates to which classical well a quantum state belongs.

To get a more precise definition of these excited-state quantum phases, we write the quantum Hamiltonian $\hat{\mathcal{H}}=\sum_{n} E_{n} \hat{P}_{n}$, where $\hat{P}_{n}$ is the projector onto the eigenspace with energy $E_{n}$. Thus, $\left[\hat{\mathcal{C}}, \hat{P}_{n}\right]=0, \forall n / E_{n}<E_{c}$, and $\left[\hat{\mathcal{C}}, \hat{P}_{n}\right] \neq 0, \forall n / E_{n}>E_{c}$. This means that $\langle\hat{\mathcal{C}}\rangle$ is conserved by any time evolution verifying that $\langle\hat{\mathcal{H}}\rangle=E<E_{c}$, but this conservation rule no longer holds if $E>E_{c}$. Hence, there exists a phase in which the expectation value of this observable must be taken into account to properly describe both equilibrium [51] and nonequilibrium [52] thermodynamics. By contrast, the other phase is characterized by standard thermodynamics.

Numerical test.-As an illustration, we perform a numerical test on a generalization of the RM and the DM. Both models account for the interaction between a monochromatic bosonic field and $N$ identical two-level atoms. The Hamiltonian reads

$\hat{\mathcal{H}}_{\alpha}=\omega \hat{a}^{\dagger} \hat{a}+\omega_{0} \hat{J}_{z}+\frac{2 \lambda}{\sqrt{N}}\left(\hat{a}^{\dagger}+\hat{a}\right) \hat{J}_{x}+\sqrt{\frac{N \omega_{0}}{2}} \alpha\left(\hat{a}^{\dagger}+\hat{a}\right)$,

where $\hat{a}^{\dagger}$ and $\hat{a}$ are the appropriate bosonic creation and annihilation operators, respectively, and $\hat{\mathbf{J}}=\left(\hat{J}_{x}, \hat{J}_{y}, \hat{J}_{z}\right)$ is an angular momentum. The total angular momentum $\hat{\mathbf{J}}^{2}$ is conserved. The dynamics of a set of $N$ identical two-level atoms is recovered with $j=N / 2$, which we use. Then, $\omega_{0}$ represents the constant splitting of the atom eigenlevels, while $\omega$ represents the frequency of the photons to which atoms are coupled by the parameter $\lambda$.

The case $\alpha=0$ is the standard RM and DM. The Hamiltonian $\hat{\mathcal{H}}_{\alpha=0}$ has a discrete $\mathbb{Z}_{2}$ symmetry, called parity, allowing to separate eigenstates according to $\hat{\Pi}\left|E_{n, \pm}\right\rangle= \pm\left|E_{n, \pm}\right\rangle, \quad$ where $\quad \hat{\Pi} \equiv \exp \left[i \pi\left(j+\hat{J}_{z}+\hat{a}^{\dagger} \hat{a}\right)\right]$ and $\hat{\mathcal{H}}_{0}\left|E_{n, \pm}\right\rangle=E_{n, \pm}\left|E_{n, \pm}\right\rangle$. If $\alpha \neq 0$, $\hat{\Pi}$ is not conserved: $\left[\hat{\mathcal{H}}_{\alpha \neq 0}, \hat{\Pi}\right] \neq 0$.

Equation (1) admits two different thermodynamic limits (TLs): (i) In the DM, the number of two-level atoms goes to infinity, $N \rightarrow \infty$, fixing $\omega_{0} / \omega<\infty$; (ii) in the RM, $\omega_{0} / \omega \rightarrow \infty$, fixing $N=1$ [48]. Thus, in the RM (DM), we set $N=1\left(\omega=\omega_{0}=1\right)$ and let $\omega_{0} / \omega(N)$ be the scaling parameter, which we denote simply $\mathcal{N}$. We use the reduced energy scale $\epsilon \equiv E /\left(\omega_{0} j\right)$. In both models, there exists a certain coupling $\lambda_{c}$ only above which ESQPTs start appearing at a certain critical energy [53].

These models fulfill the condition for the existence of the operator $\hat{\mathcal{C}}$. From their semiclassical energy surfaces [15,43-46,53] [Figs. 2(a) and 2(d)], we propose that the relevant dynamical function is $f(\mathbf{x})=q-q_{c}(\alpha, \lambda)$, where $q_{c}(\alpha, \lambda)$ is the canonical coordinate corresponding to the ESQPT critical energy. If $\alpha=0, q_{c}(0, \lambda)=0, \forall \lambda>\lambda_{c}$; if $\alpha \neq 0, q_{c}(\alpha, \lambda)$ is a complicated function [53]. Hence, $\hat{\mathcal{C}}$ takes the form

$$
\hat{\mathcal{C}}=\operatorname{sgn}\left[\hat{q}-q_{c}(\alpha, \lambda)\right]
$$

where $\hat{q}=\left(\hat{a}^{\dagger}+\hat{a}\right) / \sqrt{2}$ in the RM and $\hat{q}=\left(\hat{a}^{\dagger}+\hat{a}\right) / \sqrt{2 j}$ in the DM.

To test this hypothesis, we work with $\lambda=3 \sqrt{\omega \omega_{0}} / 2$ and two different values of the perturbation $\alpha$; in both cases, $\lambda>\lambda_{c}$, so that ESQPTs exist. We chose an initial state $|\Psi(t=0)\rangle$, with ten consecutive eigenstates equally populated. Then, we calculate the time evolution $\langle\hat{\mathcal{C}}(t)\rangle=\langle\Psi(t)|\hat{\mathcal{C}}| \Psi(t)\rangle$ for $M=100$ time steps. Next, we calculate its mean, accounting for the long-time average $\langle\hat{\mathcal{C}}\rangle=(1 / M) \sum_{i=1}^{M}\left\langle\hat{\mathcal{C}}\left(t_{i}\right)\right\rangle$. Finally, we account for the fluctuations around this value using the width $\sigma_{\mathcal{C}}^{2}=(1 / M) \sum_{i=1}^{M}\left(\left\langle\hat{\mathcal{C}}\left(t_{i}\right)\right\rangle-\overline{\langle\hat{\mathcal{C}}\rangle}\right)^{2}$. This protocol is repeated for different initial states with increasing energy values, probing different regions of the spectrum. Results are shown in Fig. 1. In the RM we work with $\mathcal{N}=300$ and $\alpha \in\{0,1 / \sqrt{3}\}$, while in the $\mathrm{DM} \mathcal{N}=40$ and $\alpha \in\{0,1 / 2\}$. We can see that $\sigma_{\mathcal{C}}^{2}$ jumps abruptly from $\sigma_{\mathcal{C}}^{2}=$ 0 to $\sigma_{\mathcal{C}}^{2}>0$ at the corresponding ESQPT criticalities in the RM for both values of $\alpha$. This means that $\hat{\mathcal{C}}$ stops being constant at this critical energy. The jump is not so abrupt in the DM, because $\mathcal{N}$ is one order of magnitude smaller. The different behavior of $\sigma_{\mathcal{C}}^{2}$ above the ESQPT in the RM and $\mathrm{DM}$ is presumably linked to their different classical degrees of freedom; however, this does not affect the results of this Letter [53].

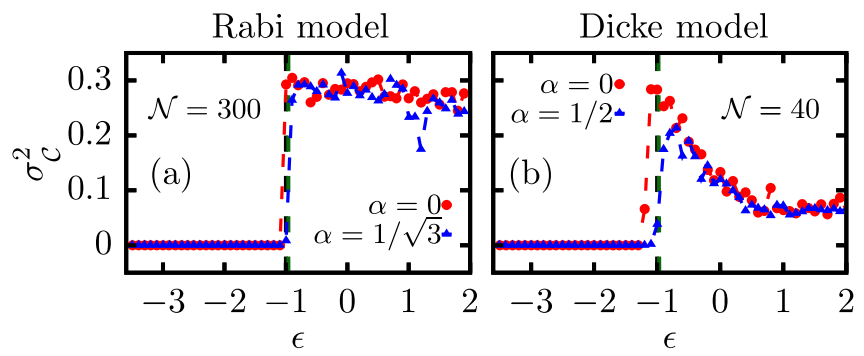

FIG. 1. Variance of the time evolution $\langle\hat{\mathcal{C}}(t)\rangle$ as a function of energy with $\lambda=3 \sqrt{\omega \omega_{0}} / 2>\lambda_{c}$ for the RM (a) and DM (b). Black (green) dashed lines mark the ESQPT critical energy for $\alpha=0(\alpha=1 / \sqrt{3}$ in the RM and $\alpha=1 / 2$ in the DM) [53]. 
Properties of the low-energy phase.-Our next step is to derive some mathematical consequences of the previous facts. We begin with the case $\alpha \neq 0$. Figure 2(e) shows the semiclassical density of states $Q(\epsilon) \equiv$ $(1 / \mathcal{N})\left[1 /(2 \pi)^{\nu}\right] \int d^{\nu} p \int d^{\nu} q \delta\left[\epsilon-H_{\alpha}(p, q)\right]$ for the RM with $\alpha=1 / \sqrt{3}$. We observe two ESQPTs, marked by (i) a logarithmic singularity at $\epsilon_{c} \approx-0.96$ and (ii) a finite jump at $\epsilon_{c_{2}} \approx-2.46$. Figure 2(d) shows the corresponding semiclassical phase space. Below $\epsilon_{c_{2}}$, there is a single connected region of constant energy curves, on the left. At $\epsilon_{c_{2}}$, a disconnected second region appears on the right. At $\epsilon_{c}$, both become connected.

Figure 2(f) shows the quantum diagonal expectation values of $\hat{\mathcal{C}}$. Our conjecture applies for $\epsilon<\epsilon_{c}$ : In this region, eigenstates of the Hamiltonian are also eigenstates of $\hat{\mathcal{C}}$, and, therefore, $\left\langle E_{n}|\hat{\mathcal{C}}| E_{n}\right\rangle= \pm 1$. But this operator also provides a description for the other ESQPT. Below $\epsilon_{c_{2}}$, all the eigenstates verify $\left\langle E_{n}|\hat{\mathcal{C}}| E_{n}\right\rangle=-1$. This means that $\hat{\mathcal{C}}$ is not necessary to account for the physics of observables in equilibrium: Any initial state is characterized by $\langle\hat{\mathcal{C}}>=$ -1 within this region, as all quantum eigenstates are attached to the left well of the classical phase space. By contrast, Fig. 2(f) also clearly shows that the conditions for the eigenstate thermalization hypothesis [60-65] are not fulfilled if $\epsilon_{c_{2}}<\epsilon<\epsilon_{c}-\left\langle E_{n}|\hat{\mathcal{C}}| E_{n}\right\rangle$ jumps abruptly from

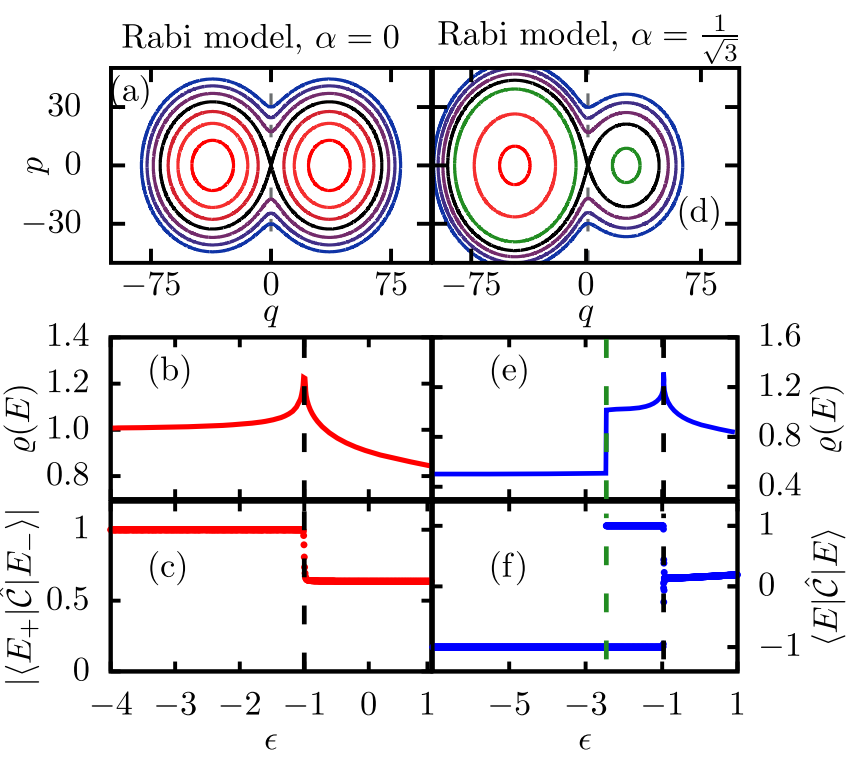

FIG. 2. (a)-(c) Semiclassical phase space, density of states, and expectation values of $\hat{\mathcal{C}}$ in quantum eigenstates for $\alpha=0$ $\left(\mathcal{N}=300\right.$ and $\left.\lambda=3 \sqrt{\omega \omega_{0}} / 2>\lambda_{c}\right)$ in the RM. (d)-(f) The same for $\alpha=1 / \sqrt{3}$. The vertical dashed lines mark the critical energies $\epsilon_{c}(\alpha=0)=-1$ and $\epsilon_{c}(\alpha=1 / \sqrt{3}) \approx-0.96$ (black) and $\epsilon_{c_{2}}(\alpha=$ $1 / \sqrt{3}) \approx-2.46$ (green). If $\alpha=0$, both wells are symmetric and at the same energy. If $\alpha \neq 0$, the second energy well is inaccessible if $\epsilon<\epsilon_{c_{2}}$. Black curves in (a) and (d) correspond to the ESQPT energy connecting both wells.
-1 to 1 . This is because quantum eigenstates can belong either to the left or to the right well in this region. Therefore, the energy is not enough to describe equilibrium thermodynamics, and additional information, given by the knowledge of $\langle\hat{\mathcal{C}}\rangle$, is also required.

If $\alpha=0$, there is an additional $\mathbb{Z}_{2}$ symmetry: $\left[\hat{\mathcal{H}}_{\alpha=0}, \hat{\Pi}\right]=0$. It can be easily shown that $\hat{\mathcal{C}}$ changes the parity of any Fock state with well-defined parity [53]. This means that $\hat{\Pi}$ and $\hat{\mathcal{C}}$ cannot be diagonalized in the same basis as they do not commute: $[\hat{\mathcal{C}}, \hat{\Pi}] \neq 0$. Hence, as $\left[\hat{P}_{n}, \hat{\Pi}\right]=\left[\hat{P}_{n}, \hat{\mathcal{C}}\right]=0$ for every energy subspace with $E_{n}<E_{c}$, there exist two different bases diagonalizing this part of the Hamiltonian, and, thus, all energy levels in this excited-state phase must be doubly degenerate. By contrast, $\hat{\Pi}$ is the only discrete $\mathbb{Z}_{2}$ symmetry if $E>E_{c}$, and, therefore, energy levels are not expected to be degenerate in that phase. This phenomenology has been observed in a large number of models displaying ESQPTs [4-6, 49,50,66-70]. As a consequence, the eigenvectors of $\hat{\mathcal{C}}$ are $\left(\left|E_{n,+}\right\rangle \pm\left|E_{n,-}\right\rangle\right) / \sqrt{2}$, and its expectation values are $\left\langle E_{n,-}|\hat{\mathcal{C}}| E_{n,+}\right\rangle= \pm 1$, due to an arbitrary sign coming from the relative phase between the degenerate eigenstates $\left|E_{n,+}\right\rangle$ and $\left|E_{n,-}\right\rangle$ [53]. This prediction is illustrated in Figs. 2(a)-2(c). The density of states in Fig. 2(b) shows that there is a single ESQPT at $\epsilon_{c}=-1$ in this case. It marks the energy at which the two equivalent wells in the classical phase space [Fig. 2(a)] become connected; below $\epsilon_{c}$, we have two disconnected, symmetric wells. Figure 2(c) clearly shows that our conjecture indeed holds below this energy: $\left|\left\langle E_{n,-}|\hat{\mathcal{C}}| E_{n,+}\right\rangle\right|=1$ for $E<E_{c}$, whereas $\left|\left\langle E_{n,-}|\hat{\mathcal{C}}| E_{n,+}\right\rangle\right| \neq 1$ for $E>E_{c}$.

Finite-size scaling.-Physically, this last case is more challenging, since we need two noncommuting discrete symmetries, $\hat{\Pi}$ and $\hat{\mathcal{C}}$, to build a complete description of thermodynamic equilibrium. Hence, we work with $\alpha=0$, which has been the object of recent experimental works [71,72], to perform a stringent test on our conjecture. As explained above, it implies energy doublets $\left|E_{n,+}-E_{n,-}\right|=0$, and also $\left|\left\langle E_{n,+}|\hat{\mathcal{C}}| E_{n,-}\right\rangle\right|=1$ if $E_{n}<E_{c}$, in the TL. To study finite systems, we define a finite-size precursor of the ESQPT as the energy $\varepsilon(\mathcal{N}, \gamma)$ above which the gap $\Delta E_{n} \equiv\left|E_{n,+}-E_{n,-}\right| /\langle s\rangle>\gamma$ or the difference $1-\left|\left\langle E_{n,+}|\hat{\mathcal{C}}| E_{n,-}\right\rangle\right|>\gamma$ for a given small bound $\gamma>0$. Here, $\langle s\rangle$ is the mean level spacing calculated within a window of ten eigenlevels around the target energy. According to our conjecture, $\lim _{\mathcal{N} \rightarrow \infty} \varepsilon(\mathcal{N}, \gamma)=\epsilon_{c}$ for any (small) $\gamma$; for finite systems, the closer the energy to the ESQPT, the larger $\mathcal{N}$ is needed to get the above indicators below a given bound [53]. In Figs. 3(a) and 3(c), we test this result with $\varepsilon(\mathcal{N}, \gamma)$ extracted from the condition on $\left|\left\langle E_{n,+}|\hat{\mathcal{C}}| E_{n,-}\right\rangle\right|$; in Figs. 3(b) and 3(d), $\varepsilon(\mathcal{N}, \gamma)$ is obtained from the condition on $\Delta E_{n}$ instead. We can clearly see that $\left|\varepsilon(\mathcal{N}, \gamma)-\epsilon_{c}\right| \propto \mathcal{N}^{-\beta}$, with $\beta>0$, in all cases, with 


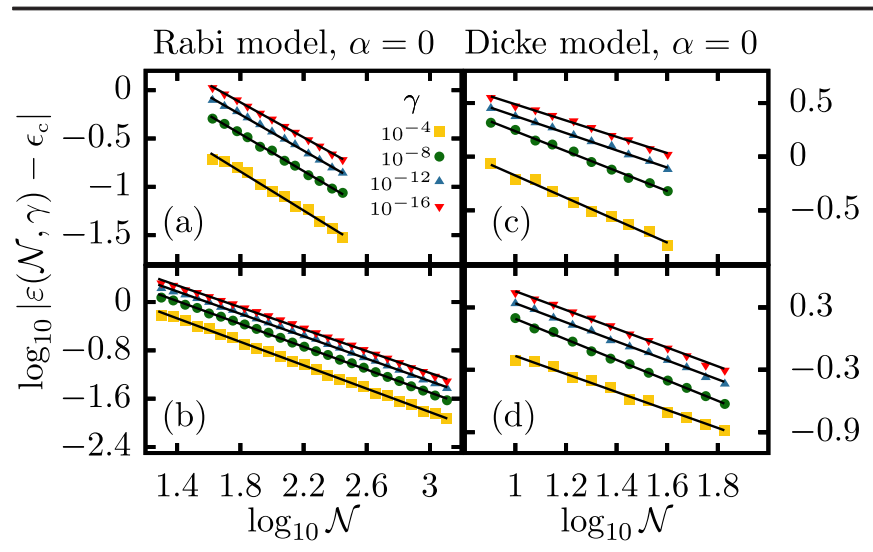

FIG. 3. Finite-size scaling of the ESQPT precursor $\varepsilon(\mathcal{N}, \gamma)$ for different bounds $\gamma$ (points), extracted from (a),(c) the expectation values of $\hat{\mathcal{C}}$ and (b),(d) eigenlevel degeneracies [53]. Solid lines represent the best linear fit to the points.

bounds ranging from $\gamma=10^{-16}$ to $\gamma=10^{-4}$. This strongly suggests that, in the TL, $\varepsilon(\mathcal{N}, \gamma) \rightarrow \epsilon_{c}$ following a power law in $\mathcal{N}$. In other words, the finite-size scaling around $\epsilon_{c}$ behaves like in standard quantum and thermal phase transitions.

Thermodynamic consequences.-Finally, we tackle the thermodynamic implications by studying the properties of observables in equilibrium in the DM with $\alpha=0$ (similar results are obtained in the RM). We start from its most general ground state at $\lambda_{\text {ini }}=\frac{3}{2} \lambda_{c}>\lambda_{c}$, given by $|\Psi(t=0)\rangle=\sqrt{p}\left|E_{\mathrm{GS},+}\right\rangle+e^{i \phi} \sqrt{1-p}\left|E_{\mathrm{GS},-}\right\rangle$, where $\hat{\mathcal{H}}_{0}\left(\lambda_{\text {ini }}\right)\left|E_{\mathrm{GS}, \pm}\right\rangle=E_{\mathrm{GS}}\left|E_{\mathrm{GS}, \pm}\right\rangle, 0 \leq p \leq 1$, and $0 \leq \phi<2 \pi$. In this state, $\langle\Psi(0)|\hat{\mathcal{C}}| \Psi(0)\rangle=2 \sqrt{p(1-p)} \cos \phi$. We then perform a quench, $\lambda_{\text {ini }} \rightarrow \lambda_{\text {fin }}$, so the time-evolved density matrix $\hat{\rho}(t) \equiv|\Psi(t)\rangle\langle\Psi(t)|$ reads $(\hbar=1)$

$$
\hat{\rho}(t)=\sum_{m, n} \sum_{k, \ell= \pm} c_{m, k} c_{n, \ell}^{*} e^{-i\left(E_{m, k}-E_{n, \ell}\right) t}\left|E_{m, k}\right\rangle\left\langle E_{n, \ell}\right|
$$

where $c_{m, k} \equiv\left\langle E_{m, k} \mid \Psi(0)\right\rangle$ [53], and all the eigenenergies and eigenstates after the quench are those of $\mathcal{H}_{0}\left(\lambda_{\text {fin }}\right), \hat{\mathcal{H}}_{0}\left(\lambda_{\text {fin }}\right)\left|E_{m, k}\right\rangle=E_{m, k}\left|E_{m, k}\right\rangle$.

First, we consider the time-evolved expectation value $\langle\hat{\mathcal{C}}(t)\rangle \equiv \operatorname{Tr}[\hat{\rho}(t) \hat{\mathcal{C}}]$. From Figs. 3(a) and 3(c), we can conclude that only the terms with $m=n$ and $k=-\ell$ contribute to $\langle\hat{\mathcal{C}}(t)\rangle$ in the TL, since $\left\langle E_{m, k}|\hat{\mathcal{C}}| E_{n, \ell}\right\rangle=$ $\pm \delta_{m, n}\left(1-\delta_{k, \ell}\right)$ if $E_{m}, E_{n}<E_{c}$; from Figs. 3(b) and 3(d), we also conclude that this contribution always remains constant and depends exclusively on the initial condition encoded in $c_{m, k},\langle\hat{\mathcal{C}}(t)\rangle=\sum_{n} c_{n,+}^{*} c_{n,-}\left\langle E_{n,+}|\hat{\mathcal{C}}| E_{n,-}\right\rangle$. Hence, the abrupt change inferred from Fig. 3 implies a change from constant to nonconstant $\langle\hat{\mathcal{C}}(t)\rangle$ at the critical energy in the TL [53].

A further consequence is that the long-time average of the time-evolved wave function in this phase [73],

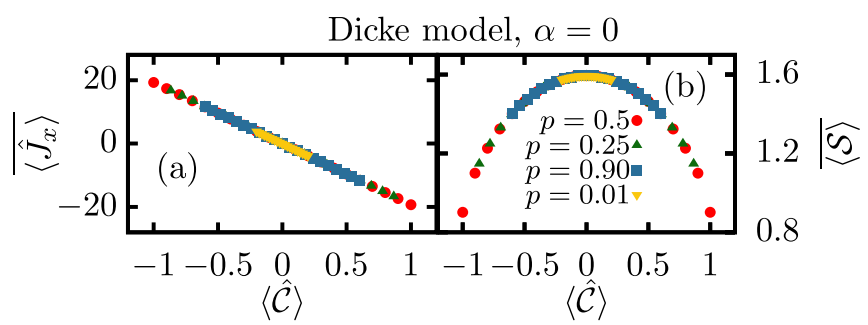

FIG. 4. Long-time averages of (a) $\hat{J}_{x}$ and (b) $\mathcal{S}$ following a quench $\lambda_{\text {ini }}=\frac{3}{2} \lambda_{c} \rightarrow \lambda_{\text {fin }}=3 \lambda_{c}$ in the DM $(\alpha=0)$ from initial states with different $p$ and $\phi$.

$\overline{\hat{\rho}}=\lim _{\tau \rightarrow \infty}(1 / \tau) \int_{0}^{\tau} d t \hat{\rho}(t)$, generally depends on both $\langle\hat{\Pi}\rangle$ and $\langle\hat{\mathcal{C}}\rangle$-not only on the energy as would be expected in the microcanonical ensemble. The longtime average of a typical observable $\hat{\mathcal{O}}$ is, therefore, $\overline{\langle\hat{\mathcal{O}}\rangle} \equiv \lim _{\tau \rightarrow \infty}(1 / \tau) \int_{0}^{\tau} d t\langle\Psi(t)|\hat{\mathcal{O}}| \Psi(t)\rangle=\operatorname{Tr}[\overline{\hat{\rho}} \hat{\mathcal{O}}] . \quad$ To explore this, we choose $\lambda_{\text {fin }}=3 \lambda_{c}$; then the energy of the nonequilibrium state is $\epsilon_{\text {fin }}=\left\langle\Psi(0)\left|\hat{\mathcal{H}}_{0}\left(\lambda_{\text {fin }}\right)\right| \Psi(0)\right\rangle /$ $\left(\omega_{0} j\right) \approx-3.15<\epsilon_{c}=-1$. We monitor the behavior of two representative observables $\hat{J}_{x}$ and $\mathcal{S}(t)$, after letting the system relax during a time $t=10^{6} \mu \mathrm{s}$, by means of $\tau=10^{3}$ equal steps, considering the realization of the DM discussed in Ref. [52]. Here, $\mathcal{S}(t)$ is the entanglement entropy: $\mathcal{S}(t) \equiv-\operatorname{Tr}\left[\hat{\rho}_{s}(t) \log \hat{\rho}_{s}(t)\right]$, where $\hat{\rho}_{s}(t)=\operatorname{Tr}_{E}|\Psi(t)\rangle\langle\Psi(t)|$, with the "environment" corresponding to the photonic radiation and the "system" being the atomic part of the Hamiltonian.

We observe in Fig. 4 that both long-time averages $\overline{\left\langle\hat{J}_{x}\right\rangle}$ and $\overline{\langle\mathcal{S}\rangle}$ crucially depend on $\langle\hat{\mathcal{C}}\rangle=\overline{\langle\hat{\mathcal{C}}\rangle}$. This means that the system reaches different equilibrium states characterized by the same energy, depending on the initial value of the coherence between parity sectors, given by the angle $\phi$. Therefore, one must take into account the expected value of $\hat{\mathcal{C}}$ to properly describe equilibrium states below the critical energy of the ESQPT, much in the same way that in the region $\epsilon_{c_{2}}<\epsilon<\epsilon_{c}$ in the case with $\alpha \neq 0$. We note that neither $\overline{\left\langle\hat{J}_{x}\right\rangle}$ nor $\overline{\langle\mathcal{S}\rangle}$ depend on $p$. Thus, they do not depend on $\langle\hat{\Pi}\rangle$ either, even though parity is an exact constant of motion in both phases. Therefore, we conclude that the role played by $\hat{\mathcal{C}}$ in thermodynamics is much more important than that of $\hat{\Pi}$.

Finally, we remark that, after a quench onto the normal phase, long-time averages depend on only the final energy $\epsilon_{\text {fin }}$ (not shown), as expected in the standard microcanonical ensemble [60].

Conclusions. - The main result of this Letter can be summarized in the following phase diagram characterizing two phases with different dynamical and thermodynamic properties.

A phase where there exists an operator $\hat{\mathcal{C}}$ with two eigenvalues, $\operatorname{Spec}(\hat{\mathcal{C}})=\{ \pm 1\}$, commuting with the 
corresponding part of the Hamiltonian, $\left[\hat{\mathcal{C}}, \hat{P}_{n}\right]=0$, $E_{n}<E_{c}$. If the system has a discrete symmetry and $[\hat{\mathcal{C}}, \hat{\Pi}] \neq 0$, then this is a broken-symmetry phase where equilibrium states are a mixture of states with broken $\hat{\Pi}$, broken $\hat{\mathcal{C}}$, or both. Furthermore, thermodynamics crucially depends on $\langle\hat{\mathcal{C}}\rangle$.

A normal phase, where $\hat{\mathcal{C}}$ is no longer a constant of motion. If there exists a discrete $\mathbb{Z}_{2}$ symmetry, then all the eigenstates of the Hamiltonian are also eigenstates of this symmetry in this phase.

Additionally, $\hat{\mathcal{C}}$ provides a description of other kind of ESQPTs, too, as exemplified by the abrupt jump in the level density of the RM and DM with $\alpha \neq 0$ and versions of the Lipkin model $[6,69,74]$.

This Letter provides a powerful framework to identify a broad class of ESQPTs dynamically, as the number of constants of motion abruptly changes at the corresponding critical energy. This should entail important consequences for nonequilibrium processes crossing an ESQPT due to the change of conserved charges $[15,52]$ and also for the steady states resulting from dynamical phase transitions $[23,75]$. A consequence of the noncommutativity of $\hat{\mathcal{C}}$ and $\hat{\Pi}$ is the possibility to build equilibrium states in which the information about both the population of each symmetric well and the quantum coherence between them is recorded.

Further research is needed to determine how to link our results with typical features of standard phase transitions, like critical slowing down. Experimental tests involving broken-symmetry equilibrium states [71,72] will play a relevant role in this endeavor.

We acknowledge financial support from Ministerio de Ciencia, Innovación y Universidades/Agencia Estatal de Investigación (MCIU/AEI/FEDER, EU) under Grant No. PGC2018-094180-B-I00.

*angelo04@ucm.es

†armando.relano@fis.ucm.es

[1] S. Sachdev, Quantum Phase Transitions (Cambridge University Press, Cambridge, England, 1999).

[2] M. A. Caprio, P. Cejnar, and F. Iachello, Excited state quantum phase transitions in many-body systems, Ann. Phys. (Amsterdam) 323, 1106 (2008).

[3] P. Stránský, M. Macek, and P. Cejnar, Excited-state quantum phase transitions in systems with two degrees of freedom: Level density, level dynamics, thermal properties, Ann. Phys. (Amsterdam) 345, 73 (2014).

[4] P. Cejnar, P. Stránský, M. Macek, and M. Kloc, Excitedstate quantum phase transitions, J. Phys. A 54 (2021) 133001 .

[5] A. Relaño, J. M. Arias, J. Dukelsky, J. E. García-Ramos, and P. Pérez-Fernández, Decoherence as a signature of an excited-state quantum phase transition, Phys. Rev. A 78, 060102(R) (2008).
[6] P. Pérez-Fernández, A. Relaño, J. M. Arias, J. Dukelsky, and J.E. García-Ramos, Decoherence due to an excitedstate quantum phase transition in a two-level boson model, Phys. Rev. A 80, 032111 (2009).

[7] P. Pérez-Fernández, P. Cejnar, J. M. Arias, J. Dukelsky, J. E. García-Ramos, and A. Relaño, Quantum quench influenced by an excited-state phase transition, Phys. Rev. A 83, 033802 (2011).

[8] L. F. Santos and F. Pérez-Bernal, Structure of eigenstates and quench dynamics at an excited-state quantum phase transition, Phys. Rev. A 92, 050101(R) (2015).

[9] C. M. Lóbez and A. Relaño, Entropy, chaos, and excitedstate quantum phase transitions in the Dicke model, Phys. Rev. E 94, 012140 (2016).

[10] F. Pérez-Bernal and L. F. Santos, Effects of excited state quantum phase transitions on system dynamics, Fortschr. Phys. 65, 1600035 (2017).

[11] M. Kloc, P. Stránský, and P. Cejnar, Quantum quench dynamics in Dicke superradiance models, Phys. Rev. A 98, 013836 (2018).

[12] W. Kopylov and T. Brandes, Time-delayed feedback control of the Dicke-Hepp-Lieb superradiant quantum phase transition, New J. Phys. 17, 103031 (2015).

[13] Q. Wang and H.T. Quan, Probing the excited-state quantum phase transition through statistics of Loschmidt echo and quantum work, Phys. Rev. E 96, 032142 (2017).

[14] L. F. Santos, M. Távora, and F. Pérez-Bernal, Excited-state quantum phase transitions in many-body systems with infinite range interaction: Localization, dynamics and bifuractions, Phys. Rev. A 94, 012113 (2016).

[15] R. Puebla, A. Relaño, and J. Retamosa, Excited-state phase transition leading to symmetry-breaking steady states in the Dicke model, Phys. Rev. A 87, 023819 (2013).

[16] R. Puebla and A. Relaño, Non-thermal excited-state quantum phase transitions, Europhys. Lett. 104, 50007 (2013).

[17] R. Puebla, A. Smirne, S. F. Huelga, and M. B. Plenio, Universal Anti-Kibble-Zurek Scaling in Fully Connected Systems, Phys. Rev. Lett. 124, 230602 (2020).

[18] V. M. Bastidas, P. Pérez-Fernández, M. Vogl, and T. Brandes, Quantum Criticality and Dynamical Instability in the Kicked-Top Model, Phys. Rev. Lett. 112, 140408 (2014).

[19] R. Puebla and A. Relaño, Irreversible processes without energy dissipation in an isolated Lipkin-Meshkov-Glick model, Phys. Rev. E 92, 012101 (2015).

[20] Q. Hummel, B. Geiger, J. D. Urbina, and K. Richter, Reversible Quantum Information Spreading in Many-Body Systems near Criticality, Phys. Rev. Lett. 123, 160401 (2019).

[21] M. A. Bastarrachea-Magnani, S. Lerma-Hernández, and J. G. Hirsch, Thermal and quantum phase transitions in atom-field systems: A microcanonical analysis, J. Stat. Mech. (2016) 093105.

[22] P. Pérez-Fernández and A. Relaño, From thermal to excitedstate quantum phase transition: The Dicke model, Phys. Rev. E 96, 012121 (2017).

[23] R. Puebla, Finite-component dynamical quantum phase transitions, Phys. Rev. B 102, 220302(R) (2020). 
[24] J. Cabedo, J. Claramunt, and A. Celi, Excited-state quantum phase transitions in spin-orbit coupled Bose gases, arXiv: 2101.08253.

[25] P. Feldmann, C. Klempt, A. Smerzi, L. Santos, and M. Gessner, Interferometric Order Parameter for Excited-State Quantum Phase Transitions in Bose-Einstein Condensates, Phys. Rev. Lett. 126, 230602 (2021).

[26] Q. Wang and F. Pérez-Bernal, Signatures of excited-state quamtum phase transitions in quantum many-body systems: Phase space analysis, arXiv:2011.11932 [Phys. Rev. E (to be published)].

[27] M. Kloc, D. Simsa, F. Hanák, P. R. Kaprálová-Zdánská, P. Stránský, and P. Cejnar, Quasiclassical approach to quantum quench dynamics in the presence of an excited-state quantum phase transition, Phys. Rev. A 103, 032213 (2021).

[28] Q. Wang and F. Pérez-Bernal, Characterizing the LipkinMeshkov-Glick model excited state quantum phase transition using dynamical and statistical properties of the diagonal entropy, Phys. Rev. E 103, 032109 (2021).

[29] Q. Wang and F. Pérez-Bernal, Probing an excited-state quantum phase transition in a quantum many-body system via an out-of-time-order correlator, Phys. Rev. A 100, 062113 (2019).

[30] P. Stránský and P. Cejnar, Classification of excited-state quantum phase transitions for arbitrary number of degrees of freedom, Phys. Lett. A 380, 2637 (2016).

[31] N. F. Zobov, S. V. Shirin, O. L. Polyansky, J. Tennyson, P.-F. Coheur, P. F. Bernath, M. Carleer, and R. Colin, Monodromy in the water molecule, Chem. Phys. Lett. 414, 193 (2005).

[32] D. Larese, F. Pérez-Bernal, and F. Iachello, Signatures of quantum phase transitions and excited state quantum phase transitions in the vibrational bending dynamics of triatomic molecules, J. Mol. Struct. 1051, 310 (2013).

[33] B. Dietz, F. Iachello, M. Miski-Oglu, N. Pietralla, A. Richter, L. von Smekal, and J. Wambach, Lifshitz and excited-state quantum phase transitions in microwave Dirac billiards, Phys. Rev. B 88, 104101 (2013).

[34] T. Tian, H.-X. Yang, L.-Y. Qiu, H.-Y. Liang, Y.-B. Yang, Y. $\mathrm{Xu}$, and L.-M. Duan, Observation of Dynamical Quantum Phase Transitions with Correspondence in an Excited State Phase Diagram, Phys. Rev. Lett. 124, 043001 (2020).

[35] J. Khalouf-Rivera, F. Pérez-Bernal, and M. Carvajal, Excited state quantum phase transitions in the bending spectra of molecules, J. Quant. Spectrosc. Radiat. Transfer 261 (2021), 107436.

[36] D. Larese and F. Iachello, A study of quantum phase transitions and quantum monodromy in the bending motion of non-rigid molecules, J. Mol. Struct. 1006, 611 (2011).

[37] M. A. Quiroz-Juárez, J. Chávez-Carlos, J. L. Aragón, J. G. Hirsch, and R. J. León-Montiel, Experimental realization of the classical Dicke model, Phys. Rev. Research 2, 033169 (2020).

[38] J. Chávez-Carlos, B. López-del-Carpio, M. A. BastarracheaMagnani, P. Stránský, S. Lerma-Hernández, L. F. Santos, and J. G. Hirsch, Quantum and Classical Lyapunov Exponents in Atom-Field Interaction Systems, Phys. Rev. Lett. 122, 024101 (2019).

[39] M. A. Bastarrachea-Magnani, B. López-del-Carpio, J. Chávez-Carlos, S. Lerma-Hernández, and J. G. Hirsch,
Delocalization and quantum chaos in atom-field systems, Phys. Rev. E 93, 022215 (2016).

[40] I. I. Rabi, On the process of space quantization, Phys. Rev. 49, 324 (1936).

[41] I. I. Rabi, Space quantization in a gyrating magnetic field, Phys. Rev. 51, 652 (1937).

[42] R. H. Dicke, Coherence in spontaneous radiation processes, Phys. Rev. 93, 99 (1954).

[43] M. A. Bastarrachea-Magnani, S. Lerma-Hernández, and J. G. Hirsch, Comparative quantum and semi-classical analysis of Atom-Field Systems. I. Density of states and excited-state quantum phase transitions, Phys. Rev. A 89, 032101 (2014).

[44] M. A. Bastarrachea-Magnani, S. Lerma-Hernández, and J. G. Hirsch, Comparative quantum and semiclassical analysis of atom-field systems. II. Chaos and regularity, Phys. Rev. A 89, 032102 (2014).

[45] A. Relaño, M. A. Bastarrachea-Magnani, and S. LermaHernández, Approximated integrability of the Dicke model, Europhys. Lett. 116, 50005 (2016).

[46] M. A. Bastarrachea-Magnani, A. Relaño, S. LermaHernández, B. López-del-Carpio, J. Chávez-Carlos, and J. G. Hirsch, Adiabatic invariants for the regular region of the Dicke model, J. Phys. A 50, 144002 (2017).

[47] T. Brandes, Excited-state quantum phase transitions in Dicke superradiance models, Phys. Rev. E 88, 032133 (2013).

[48] R. Puebla, M.-J. Hwang, and M. B. Plenio, Excited-state quantum phase transition in the Rabi model, Phys. Rev. A 94, 023835 (2016).

[49] A. Relaño, J. Dukelsky, P. Pérez-Fernández, and J. M. Arias, Quantum phase transitions of atom-molecule Bose mixtures in a double-well potential, Phys. Rev. E 90, 042139 (2014).

[50] J. E. García-Ramos, P. Pérez-Fernández, and J. M. Arias, Excited-state quantum phase transitions in a two-fluid Lipkin model, Phys. Rev. C 95, 054326 (2017).

[51] Y. Guryanova, S. Popescu, A. J. Short, R. Silva, and P. Skrzypczyk, Thermodynamics of quantum systems with multiple conserved quantities, Nat. Commun. 7, 12049 (2016).

[52] J. Mur-Petit, A. Relaño, R. A. Molina, and D. Jaksch, Revealing missing charges with generalised quantum fluctuation relations, Nat. Commun. 9, 2006 (2018).

[53] See Supplemental Material at http://link.aps.org/ supplemental/10.1103/PhysRevLett.127.130602 for a brief review of the semiclassical analog of Eq. (1), mathematical details of $\hat{\mathcal{C}}$, supplemental figures of the finite-size scaling, an example of the time-evolved expectation values $\langle\hat{\mathcal{C}}(t)\rangle$, and information about the initial states used and the populations after the quench, which includes Refs. [54-59].

[54] C. Emary and T. Brandes, Quantum Chaos Triggered by Precursors of a Quantum Phase Transition: The Dicke Model, Phys. Rev. Lett. 90, 044101 (2003).

[55] C. Emary and T. Brandes, Chaos and the quantum phase transition in the Dicke model, Phys. Rev. E 67, 066203 (2003).

[56] P. Pérez-Fernández, A. Relaño, J. M. Arias, P. Cejnar, J. Dukelsky, and J.E. García-Ramos, Excited-state phase transition and onset of chaos in quantum optical models, Phys. Rev. E 83, 046208 (2011). 
[57] M.-J. Hwang, R. Puebla, and M. B. Plenio, Quantum Phase Transition and Universal Dynamics in the Rabi Model, Phys. Rev. Lett. 115, 180404 (2015).

[58] S. Pilatowsky-Cameo, D. Villaseńor, M. A. BastarracheaMagnani, S. Lerma-Hernández, L. F. Santos, and J.G. Hirsch, Quantum scarring in a spin-boson system: Fundamental families of periodic orbits, New J. Phys. 23, 033045 (2021).

[59] J. D. Roberts, Linear model reduction and solution of the algebraic Riccati equation by use of the sign function, Int. J. Control 32, 677 (1980).

[60] L. D'Alessio, Y. Kafri, A. Polkovnikov, and M. Rigol, From quantum chaos and eigenstate thermalization to statistical mechanics and thermodynamics, Adv. Phys. 65, 239 (2016).

[61] H. Tasaki, From Quantum Dynamics to the Canonical Distribution: General Picture and a Rigorous Example, Phys. Rev. Lett. 80, 1373 (1998).

[62] M. Rigol, V. Dunjko, and M. Olshanii, Thermalization and its mechanism for generic isolated quantum systems, Nature (London) 452, 854 (2008).

[63] P. Reimann, Eigenstate thermalization: Deutsch's approach and beyond, New J. Phys. 17, 055025 (2015).

[64] J. M. Deutsch, Eigenstate thermalization hypothesis, Rep. Prog. Phys. 81, 082001 (2018).

[65] M. Srednicki, Chaos and quantum thermalization, Phys. Rev. E 50, 888 (1994).

[66] M. Sindelka, L. F. Santos, and N. Moiseyev, Excited-state quantum phase transitions studied from a non-Hermitian perspective, Phys. Rev. A 95, 010103(R) (2017).

[67] P. Cejar, M. Macek, S. Heinze, J. Jolie, and J. Dobes, Monodromy and excited-state quantum phase transitions in integrable systems: Collective vibrations of nuclei, J. Phys. A 39, L515 (2006).

[68] P. Cejnar, S. Heinze, and M. Macek, Coulomb Analogy for Non-Hermitian Degeneracies near Quantum Phase Transitions, Phys. Rev. Lett. 99, 100601 (2007).

[69] P. Ribeiro, J. Vidal, and R. Mosseri, Exact spectrum of the Lipkin-Meshkov-Glick model in the thermodynamic limit and finite-size corrections, Phys. Rev. E 78, 021106 (2008).

[70] J. Khalouf-Rivera, M. Carvajal, and F. Pérez-Bernal, Quantum fidelity susceptibility in excited state quantum phase transitions: Application to the bending spectra of nonrigid molecules, arXiv:2102.12335.

[71] M.-L. Cai, Z.-D. Liu, W.-D. Zhao, Y.-K. Wu, W.-X. Mei, Y. Jiang, L. He, X. Zhang, Z.-C. Zhou, and L.-M. Duan, Observation of a quantum phase transition in the quantum Rabi model with a single trapped ion, Nat. Commun. 12, 1126 (2021).

[72] K. Baumann, R. Mottl, F. Brennecke, and T. Esslinger, Exploring Symmetry Breaking at the Dicke Quantum Phase Transition, Phys. Rev. Lett. 107, 140402 (2011).

[73] P. Reimann, Foundations of Statistical Mechanics under Experimentally Realistic Conditions, Phys. Rev. Lett. 101, 190403 (2008).

[74] H. Lipkin, N. Meshkov, and A. Glick, Validity of manybody approximation methods for a solvable model: (I). Exact solutions and perturbation theory, Nucl. Phys. 62, 188 (1965).

[75] R. J. Lewis-Swan, S. R. Muleady, D. Barberena, J. J. Bollinger, and A.M. Rey, Characterizing the dynamical phase diagram of the Dicke model via classical and quantum probes, Phys. Rev. Research 3, L022020 (2021). 